\title{
Interpol: An R package for preprocessing of protein sequences
}

\author{
Dominik Heider ${ }^{*}$ and Daniel Hoffmann
}

\author{
* Correspondence: dominik. \\ heider@uni-due.de \\ Department of Bioinformatics, \\ Center for Medical Biotechnology, \\ University of Duisburg-Essen, \\ Universitaetsstr. 2, 45141 Essen, \\ Germany
}

\begin{abstract}
Background: Most machine learning techniques currently applied in the literature need a fixed dimensionality of input data. However, this requirement is frequently violated by real input data, such as DNA and protein sequences, that often differ in length due to insertions and deletions. It is also notable that performance in classification and regression is often improved by numerical encoding of amino acids, compared to the commonly used sparse encoding.

Results: The software "Interpol" encodes amino acid sequences as numerical descriptor vectors using a database of currently 532 descriptors (mainly from AAindex), and normalizes sequences to uniform length with one of five linear or non-linear interpolation algorithms. Interpol is distributed with open source as platform independent R-package. It is typically used for preprocessing of amino acid sequences for classification or regression.

Conclusions: The functionality of Interpol widens the spectrum of machine learning methods that can be applied to biological sequences, and it will in many cases improve their performance in classification and regression.
\end{abstract}

\section{Findings}

Machine learning techniques have been widely applied to biological sequences to gain insights into biological function, for instance Rost and Sander [1], Dubchak et al. [2], Karchin et al. [3] and Nielsen et al. [4]. Nanni and Lumini [5] have found improved performance of classifiers based on numerically encoded amino acid sequences as compared to classifiers based on the typically used standard orthonormal representation, i.e. a vector containing twenty indicator variables (one for each amino acid) for each sequence position, resulting in a matrix containing the amino acid distributions for each position in the input sequence. For numerical encoding, each amino acid (or nucleotide) of a sequence is mapped to a numerical descriptor value, such as hydropathy [6], molecular weight, or isoelectric point.

One major limitation of almost all machine learning algorithms is the fixed input dimension, making these algorithms incapable of handling data which varies in its dimension. This is unsuitable for many biological applications as there are often sequence deletions and insertions.

We have developed a preprocessing approach for machine learning that combines the use of numerical descriptor values with a normalization of sequences to a fixed length by numerical interpolation. This procedure has already been applied to

\section{() Biomed Central}


coreceptor usage prediction in HIV-1 [7], functional protein classification [8,9], and HIV-1 drug resistance prediction [10] were it led to marked improvements of prediction performance. Although many machine learning algorithms are available as software, no package for the described preprocessing of amino acid sequences is available to date. We have therefore developed Interpol, a flexible and easy to use open source package for the statistical language R http://www.r-project.org/. Currently, Interpol provides encoding of amino acid sequences with 531 different numerical descriptors from the AAindex database [11] and one additional empirical descriptor. Moreover, it allows normalization of encoded sequences to a specific length with five different linear or non-linear interpolation procedures.

Interpol is included in the Comprehensive R Archive Network (CRAN) and can be directly downloaded and installed by using the following $\mathrm{R}$ command:

install.packages ("Interpol")

In the following example, we introduce Interpol's two commands AAdescriptor and Interpol applied to a set consisting of 1351 HIV-1 V3 loop sequences from Dybowski et al. [7] for the prediction of coreceptor usage (see also Table 1). After loading the set of sequences, the first V3 sequence is encoded using the AAdescriptor command:

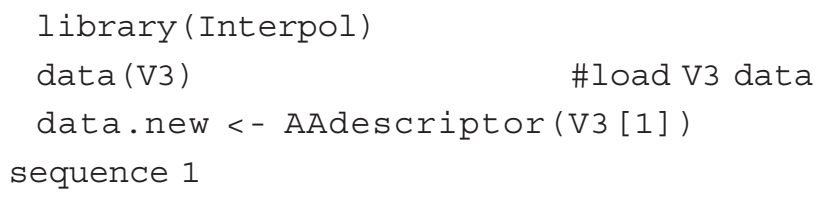

Optional parameters are the applied descriptor (default descriptor $=151$, i.e. the hydropathy scale of Kyte and Doolittle [6]) and an interval normalization (default normalize $=0$, i.e. no normalization). The list of available descriptors can be found in data (list).

After encoding the amino acid sequence as numerical vector, it can be normalized to a specific length for subsequent classification. In our example, the V3 sequence lengths vary between 33 and 38 amino acids due to deletions or insertions. The following commands translate the amino acid sequences into numerical sequences using the hydropathy descriptor, and then normalize the sequences to a fixed length of 35 :

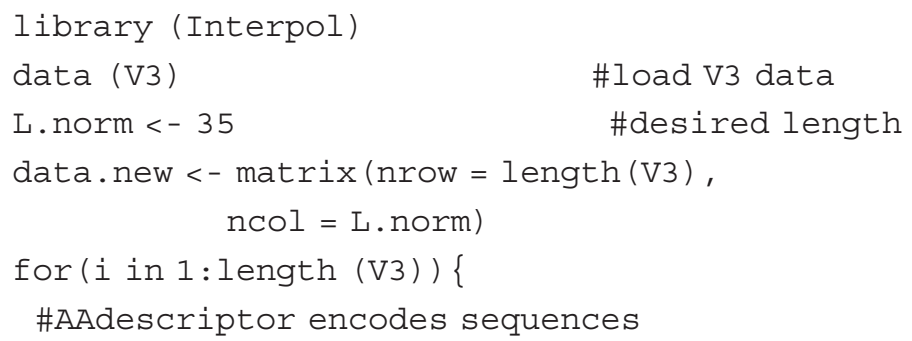

Table 1 Method overview

\begin{tabular}{lll}
\hline command & parameters & information \\
\hline AAdescriptor & data & amino acid sequence \\
& descriptor (optional) & $1-532 ;$ default $=151[6]$ \\
& normalize (optional) & $0:$ no; $1:[-1,1] ; 2:[0,1] ;$ default $=0$ \\
\hline Interpol & data & encoded amino acid sequence \\
& dims & desired length \\
& method (optional) & default = linear, spline, periodic, natural, fmm \\
\hline
\end{tabular}




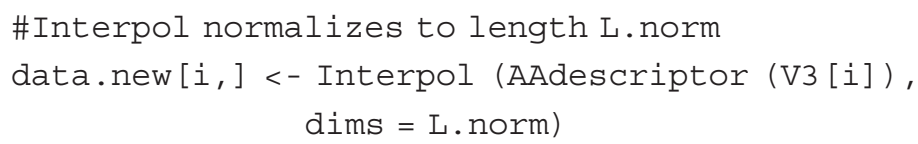

Sequence 782 in the V3 dataset has a length of 38 amino acids. In the following example the code for normalization from 38 to 35 amino acids, and for visualization of the interpolation is demonstrated (see Figure 1):
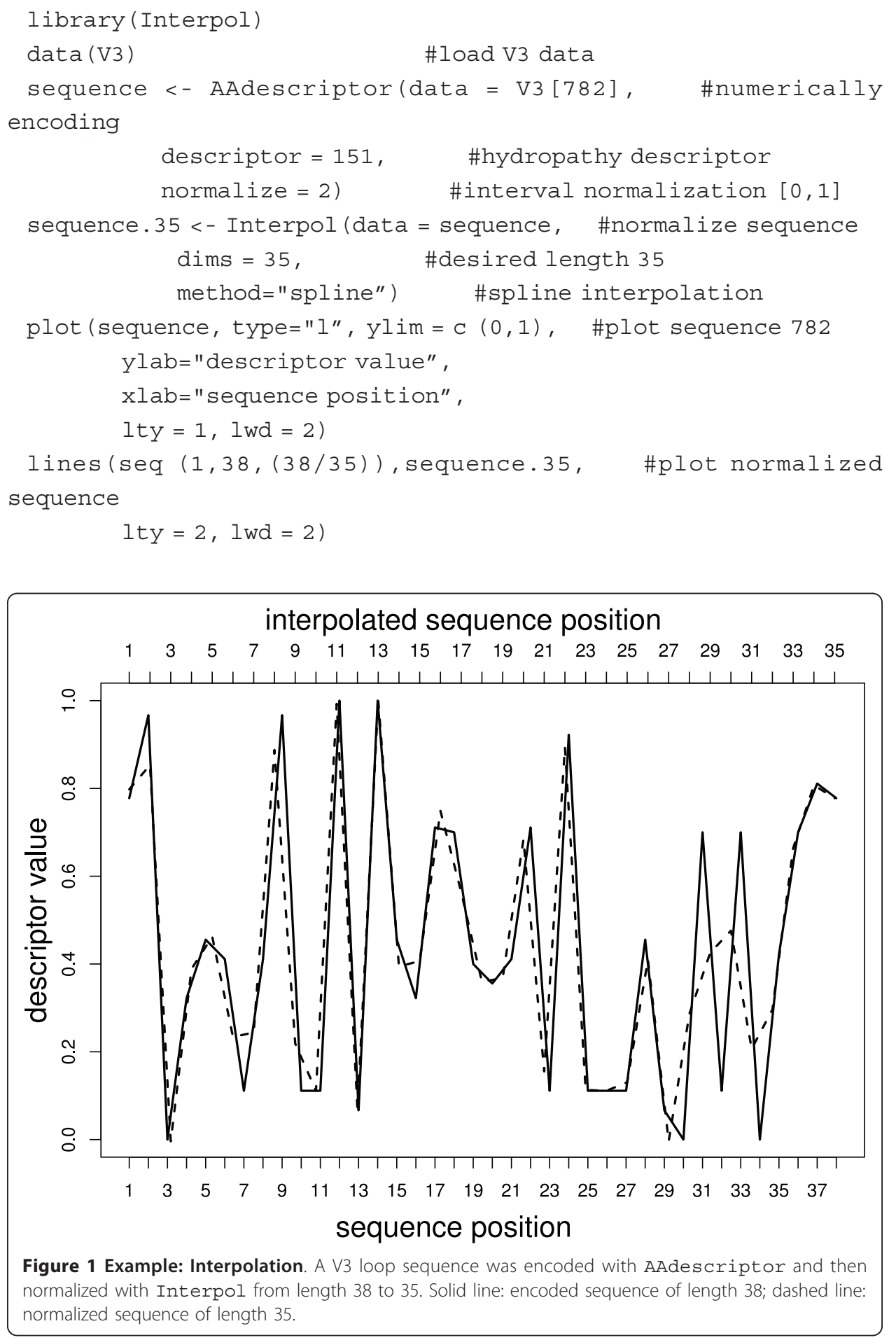


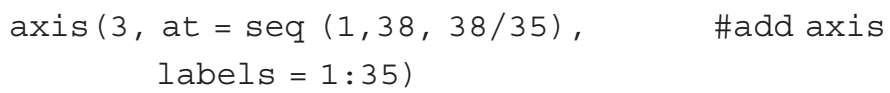

The optional parameter method can be one of linear, spline, natural, periodic or fmm (default method = linear). The linear interpolation connects two data points $\left(x_{0}, y_{0}\right)$ and $\left(x_{1}, y_{1}\right)$ with a straight line. The cubic spline interpolation uses piecewise cubic polynomials instead of a straight line. The spline interpolation of Forsythe [12] builds a cubic spline interpolation with the cubic passing exactly through the four points at each end of a sample. The periodic spline interpolation fulfills periodic boundary conditions, i.e. the spline curve has the same first and second derivative at its endpoints. The natural spline interpolation fulfills the natural boundary conditions.

The command help (package = Interpol) gives an overview of the Interpol package and the included methods and data. Descriptions for the AAdescriptor and the Interpol commands can be obtained by help (AAdescriptor) and help (Interpol), respectively.

In the following examples we demonstrate the use of the Interpol package for the prediction of the coreceptor usage of HIV-1 according to Dybowski et al. [7] based on sequences of the V3-region of the HIV-1 protein gp120. V3 is the main determinant of coreceptor usage, i.e. it determines which of the cellular coreceptors CCR5 or CXCR4 is used by HIV-1 for cell entry. Classification of V3-sequences with respect to coreceptor usage is important for therapy and prognosis. Since V3 is variable in length, many classification algorithms are not applicable. We therefore first apply AAdescriptor and Interpol to numerically encode V3-sequences and to normalize them to a fixed length. We then apply for classification random forests [13] implemented in the randomForest package, and for performance measurement the area under the receiver operating characteristics curve (AUC) implemented in the ROCR package [14] of $\mathrm{R}$ according to Dybowski et al. [7]. Note that Interpol is independent of the classification method applied, and could be also used with artificial neural networks (as in R-package neuralnet), support vector machines (as in R-package kernlab) [15], etc.

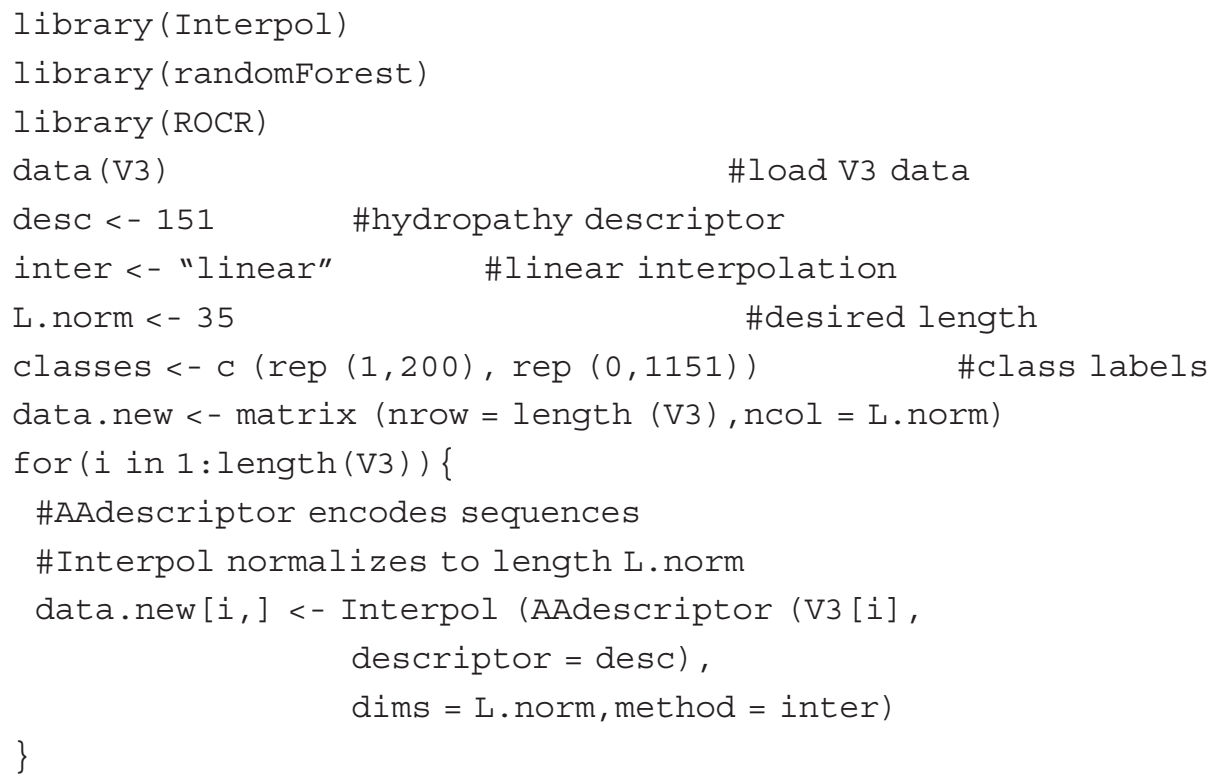


forest

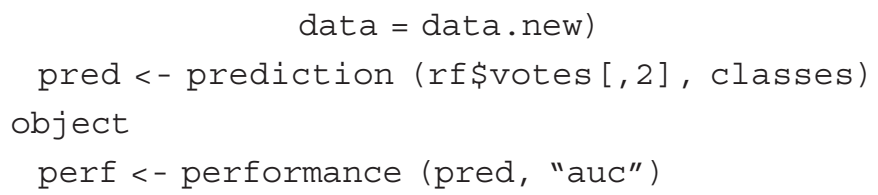

Using the Interpol package, it is very easy to retrieve and compare the performance of different descriptors, e.g. hydrophobicity and net charge, and different interpolation methods (e.g. linear and spline interpolation), by just changing line desc <- 151 to desc <- 146 and inter <- "linear" to inter <- "spline", respectively, in the above code (see also Figure 2). The complete list of descriptors can be found on the help pages (help (Interpol)) and in data (list). Note that the above code somewhat overestimates the true performance as it does not include the leave-onepatient-out scheme used by Dybowski et al. [7].

There are several potential limitations of the Interpol method for protein classification. First, normalizing to lengths of less than $50 \%$ of the original sequence length will in general lead to loss of information. Thus, we suggest to stretch short sequences to a certain length instead of squeezing longer sequences. However, stretching can also cause problems as the normalized sequence space has a higher dimension and thus classification is more prone to overfitting. A more general limitation of normalization is that in some cases the sequence length itself can carry some information. For instance, classifying sequences of huntingtin protein [16] for induction of Huntington's disease critically relies on the length of a Glutamine repeat, an information that can be partly lost in sequence normalization.

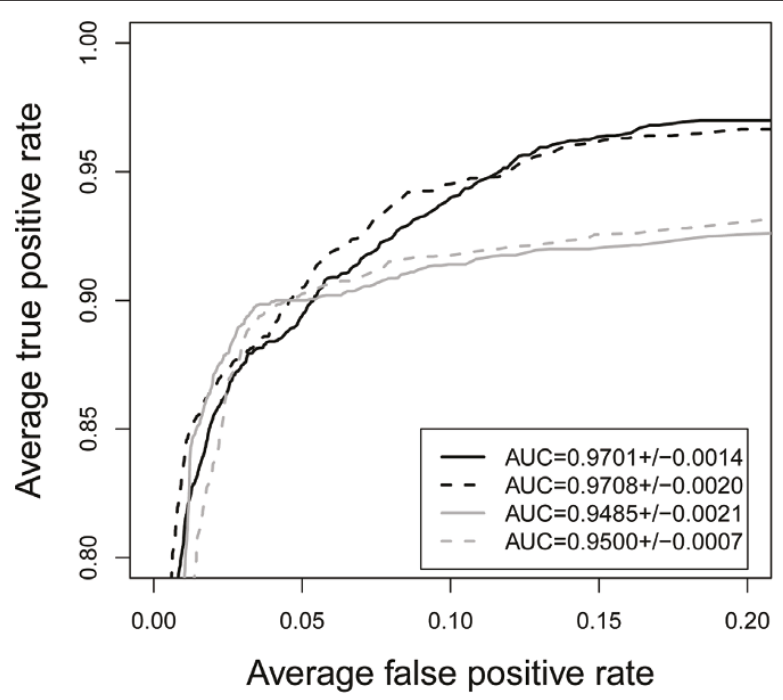

Figure 2 Example: ROC curves. Comparison of prediction performance based on different descriptors and interpolation methods implemented in Interpol and visualized with ROCR [14]. black: hydropathy (descriptor = 151); grey: net charge (descriptor = 146); solid line: linear interpolation; dashed line: spline interpolation. 


\section{Availability and requirements}

- Project name: Interpol

- Project home page (CRAN): http://cran.r-project.org/web/packages/Interpol/

- Operating system (s): Platform independent

- Programming language: $\mathrm{R}(\geq 2.10 .0)$

- License: GPL $(\geq 2)$

- Any restrictions to use by non-academics: none

\section{Acknowledgements}

We thank the reviewers for their fruitful suggestions. Funding by Deutsche Forschungsgemeinschaft TRR60/A6 is gratefully acknowledged.

\section{Authors' contributions}

$\mathrm{DH}^{*}$ has implemented and tested the software, and drafted the manuscript. DH has revised the manuscript. All authors read and approved the final manuscript.

\section{Competing interests}

The authors declare that they have no competing interests.

Received: 27 March 2011 Accepted: 17 June 2011 Published: 17 June 2011

\section{References}

1. Rost B, Sander C: Combining evolutionary information and neural networks to predict protein secondary structure. Proteins 1994, 19:55-72

2. Dubchak I, Muchnik I, Holbrook SR, Kim SH: Prediction of protein folding class using global description of amino acid sequence. Proc Natl Acad Sci 1995, 92:8700-8704.

3. Karchin R, Karplus K, Haussler D: Classifying G-protein coupled receptors with support vector machines. Bioinformatics 2002, 18:147-150.

4. Nielsen M, Lundegaard C, Worning P, Lauemøller SL, Lamberth K, Buus S, Brunak S, Lund O: Reliable prediction of T-cell epitopes using neural networks with novel sequence representations. Protein Sci 2003, 12(5):1007-1017.

5. Nanni L, Lumini A: A new encoding technique for peptide classification. Expert Systems with Applications 2011, 38(4):3185-3191.

6. Kyte J, Doolittle R: A simple method for displaying the hydropathic character of a protein. J Mol Biol 1982, 157:105-132

7. Dybowski JN, Heider D, Hoffmann D: Prediction of co-receptor usage of HIV-1 from genotype. PLoS Comput Biol 2010, 6(4):e1000743.

8. Heider D, Appelmann J, Bayro T, Dreckmann W, Held A, Winkler J, Barnekow A, Borschbach M: A computational approach for the identification of small GTPases based on preprocessed amino acid sequences. Technology in Cancer Research and Treatment 2009, 8(5):333-342.

9. Heider D, Hauke S, Pyka M, Kessler D: Insights into the classification of small GTPases. Advances and Applications in Bioinformatics and Chemistry 2010, 3:15-24.

10. Heider D, Verheyen J, Hoffmann D: Machine learning on normalized protein sequences. BMC Research Notes 2011, 4:94.

11. Kawashima S, Pokarowski P, Pokarowska M, Kolinski A, Katayama T, Kanehisa M: AAindex: amino acid index database, progress report 2008. Nucleic Acids Res 2008, , 36 Database: D202-D205.

12. Forsythe GE: Computer Methods for Mathematical Computations Prentice Hall; 1977.

13. Breiman L: Random Forests. Machine Learning 2001, 45:5-32.

14. Sing T, Sander O, Beerenwinkel N, Lengauer T: ROCR: visualizing classifier performance in R. Bioinformatics 2005, 21(20):3940-3941.

15. Karatzoglou A, Smola A, Hornik K, Zeileis A: kernlab - An S4 Package for Kernel Methods in R. Journal of Statistical Software 2004, 11(9):1-20.

16. Walker FO: Huntington's disease. Lancet 2007, 369(9557):218-228.

doi:10.1186/1756-0381-4-16

Cite this article as: Heider and Hoffmann: Interpol: An R package for preprocessing of protein sequences. BioData Mining 2011 4:16. 